\title{
The dragonfly Libellula quadrimaculata (Odonata: Libellulidae) makes optimal use of the dorsal fovea of the compound eyes during perching
}

\author{
Manuela SAUSENG ${ }^{1 *}$, Maria-AnNa PABST ${ }^{2}$ and Karl KRAL ${ }^{1}$ \\ ${ }^{1}$ Institute of Zoology, Karl-Franzens-University Graz, A-8010 Graz, Austria \\ ${ }^{2}$ Institute of Histology and Embryology, Karl-Franzens-University Graz, A-8010 Graz, Austria
}

Key words. Odonates, perching, compound eye, dorsal fovea, vision, visual ecology

\begin{abstract}
We studied visual orientation and perching behaviour of a territorial libellulid dragonfly species, Libellula quadrimaculata. The studies were performed during sunny, cloudless conditions at a pond in southern Styria, Austria, from May to July of 2001 and 2002. Individual males were observed for periods of 3 to 4 weeks.

We measured dragonfly's horizontal orientation relative to the solar azimuth, and vertical orientation relative to the solar altitude. The measurements indicated that the males had a favourable view of the sky during perching. In addition, the relative amounts of ultraviolet (UV) and blue-violet radiation in scattered light (not direct sunlight) were calculated for the whole sky and for the section of the sky viewed by the fovea. Our results show that the dorsal fovea is directed preferentially toward a section of the sky away from the sun, with less radiation but a higher UV and blue-violet saturation.

The present findings fit in well with the hypothesis, based on optical and physiological data, that the fovea, which is sensitive only to blue and UV radiation, is optimally suited to the detection of small, rapidly flying insects against the blue sky. The findings supply the first behavioural correlates of this foveal specialisation.
\end{abstract}

\section{INTRODUCTION}

The dorsal region of the compound eyes of many insects contains a fovea which exhibits not only high spatial resolution but also a high degree of sensitivity to short-wave radiation (for review see Wehner, 1981; Land, 1989, 1997; Kral, 2002). In the case of dragonflies, and particularly in the case of perching libellulids, this type of dorsal specialisation is especially pronounced (see e.g. Horridge, 1978; Laughlin \& McGinness, 1978; Sherk, 1978; Armett-Kibel \& Meinertzhagen, 1983; Meyer \& Labhart, 1993; Labhart \& Nilsson, 1995). The dorsal region of the eye of perching libellulids has facets which are considerably larger than those in the ventral region with a greater radius of curvature. In addition the ommatidia, and hence the light-sensitive rhabdomeres of the foveal photoreceptor cells, are significantly longer. There are variations in the size of the interommatidial angle within the dorsal region of the eye, due to the presence of the dorsal fovea. In the extra-foveal area, which forms by far the largest part of the dorsal region of the eye, the interommatidial angles are similar to those in the ventral region of the eye. However, in the dorsal fovea, facet diameters reach a maximum value, rhabdomeres attain their greatest length, and interommatidial angles have values below $0.5^{\circ}$. Thus, from a purely morphological point of view the dorsal fovea has the highest light efficiency and the highest resolution of the entire eye (for review see Land, 1989; Labhart \& Nilsson, 1995).

Furthermore, in perching libellulids, the specialisation of the dorsal region of the eye with regard to spectral sen- sitivity is also particularly pronounced (Labhart \& Nilsson, 1995). Due to its screening pigments the dorsal region of the eye appears yellow or orange, whereas the ventral region of the eye appears dark. In Libellula quadrimaculata this dorsal-ventral delineation is clearly visible.

The boundary between the two regions of the eye is sharp, corresponding to the morphological boundary. Electrophysiological investigations of Sympetrum (Labhart \& Nilsson, 1995) show that, in the dorsal region of the eye, two of the nine photoreceptor cells of each ommatidium are UV-sensitive, while the remaining seven are sensitive to blue light. Labhart \& Nilsson (1995) suggest that, due to the absorption properties of the yellow or orange screening pigment with respect to incident light (causing short-wave radiation to be absorbed, while letting through longer wave radiation that increases the rate of conversion of metarhodopsin to rhodopsin), the rhodopsin concentration of photopigments sensitive to shortwave radiation is kept at a high level, so that the photoreceptor cells remain maximally sensitive even when exposed to radiation (for review see Stavenga, 1989). This screening pigment mechanism reduces acceptance angles to as little as $0.3^{\circ}$, since light to which the photoreceptor cells are sensitive is absorbed. The screening pigment thus channels the short-wave radiation in such a way that high resolution is maintained and images are sharply focused (for review see Land, 1997; Kral, 2002). In the ventral region of the eye the maximum sensitivity of the photoreceptor cells lies primarily in the long-wave region of the spectrum. By means of intracellular recordings, UV, blue, green and yellow/orange pho-

\footnotetext{
* Corresponding author. E-mail: manuela.sauseng@stud.uni-graz.at (M. Sauseng)
} 

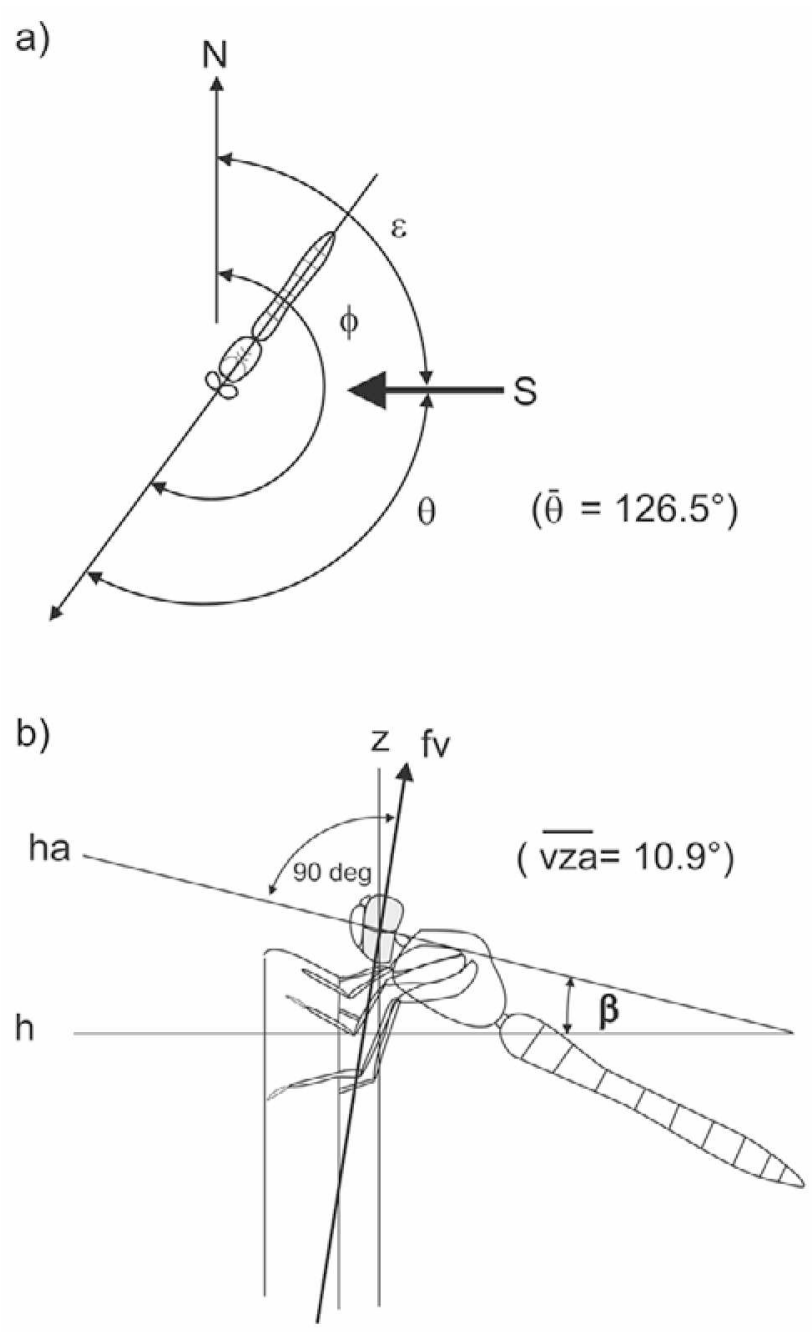

Fig. 1. Diagrams illustrating the measurement of a) the yaw angle $\phi$ and b) the pitch angle $\beta$ of the head of a Libellula quadrimaculata male maintaining an active sit-and-wait ambush posture. $\varepsilon$, solar azimuth; $\theta=$ difference between yaw angle $(\phi)$ and solar azimuth (e) (mean value of $\theta$ is shown, see Results). Diagram b) also illustrates the calculation of the line of sight of the dorsal fovea ( $\mathrm{fv}$, foveal view); vza, vertical viewing angle of the dorsal fovea $\left(\beta+90^{\circ}\right)$ relative to the zenith, i.e. the viewer zenith angle (mean value of vza is shown, see Results); h, horizontal line; vertical line points to zenith, $\mathrm{z}$; ha, head axis.

toreceptor cells have been identified in Sympetrum (Meinertzhagen et al., 1983; Yang \& Osorio, 1991; for review see Kral, 1987).

Based upon these morphological, optical and physiological characteristics, it has been postulated that the dorsal fovea of the compound eyes of perching libellulids is optimally suited to the detection of small, rapidly flying insects against the blue sky (see e.g. MazokhinPorshnyakov, 1959; Land, 1997). However, to a large extent behavioural correlates are lacking. The present study therefore addresses the question: Do perching male dragonflies orient the dorsal fovea precisely in the direction in which optimal visual background conditions for the detection of prey prevail?

\section{MATERIALS AND METHODS}

\section{Experimental animals and study site}

Field observations were recorded for eight mature males of an extremely territorial and aggressive, diurnal dragonfly species, the four-spotted chaser, Libellula quadrimaculata Linnaeus, 1758 (Odonata: Anisoptera, Libellulidae). This proved to be an ideal experimental animal because of its abundance and relatively long flying season. The studies were carried out from May to July in the summers of 2001 and 2002. The study site was a pond with natural perches, surrounded by bushes, trees, areas of open meadow, and rising ground. The pond had an area of approximately $15.6 \mathrm{~m}^{2}$ and was located in the East Styrian hill country near Graz, Austria $\left(47^{\circ} 10^{\prime} \mathrm{N}, 15^{\circ} 32^{\prime} \mathrm{E}\right)$. Observations were made only in sunny, cloudless conditions, when there was little or no wind (usually $<1 \mathrm{~ms}^{-1}$ ) and with air temperatures above $18^{\circ} \mathrm{C}$, since only under these conditions could perching dragonflies be observed (see e.g. Belyshev, 1967). During the course of the observations, we took care not to disturb the natural behaviour of the experimental animals in any way. Individual males were identified by the distinctive appearance of their spots or by means of other morphological characteristics.

\section{Behavioural field studies}

Measurement of yaw and pitch angles of the perching dragonfly relative to the position of the sun. During the course of the day, from 0800 to 1800 hours, each time a male dragonfly landed on the perch, measurements were made of the yaw (horizontal) angle $\phi$ and the pitch (vertical) angle $\beta$ after the dragonfly had oriented itself (Fig. 1).

The yaw angle $\phi$ of the dragonfly was determined relative to the solar azimuth $\varepsilon$ (Fig. 1a). Since a digital Fuji FP 4900 Zoom camera was aligned at right angles to the longitudinal axis of the dragonfly (real length of longitudinal axes was used as reference), the alignment of the camera was determined relative to the polar co-ordinates, measured by means of a magnetic compass, and $90^{\circ}$ was added to find the yaw angle $\phi$ of the experimental animal. In addition, the animals direction of view was considered directly by the magnetic compass.

In order to determine the pitch angle $\beta$ relative to the vertical, the dragonflies were photographed directly from the side (Fig. 1b). To indicate the vertical axis a plumb line was used consisting of a piece of metal tied to a thin white thread attached to the perch.

\section{Optical calculations}

Calculation of the line of sight of the dorsal fovea of the perching dragonfly. The size and position of the dorsal fovea of the compound eyes were determined on the basis of semithin plastic-embedded sections of an eye fixed in 3\% glutaraldehyde and $2 \% \mathrm{OsO}_{4}$, as well as on the basis of scanning electron micrographs and pseudopupil measurements of living eyes (see Eggenreich \& Kral, 1990). It was found that the dorsal fovea lies approximately $90^{\circ}$ above the equator of the eye (defined by the bases of the antennae). The visual field of the dorsal fovea is centered around the vertical axis of the head. Accordingly, the vertical line of sight of the dorsal fovea was calculated by adding $90^{\circ}$ to the pitch angle $\beta$, which corresponds to the line of sight of the ommatidia at the equator of the eye. The horizontal line of sight was calculated from the yaw angle $\phi$ (Fig. 1).

Measuring spectral sensitivity of the dorsal fovea. The spectral sensitivity values of the dorsal fovea of the compound eyes were determined from electroretinogram (ERG) recordings. The insect was anaesthetised with chlorethyl and then attached to a holder with a cauteriser, using a mixture of wax and resin, 

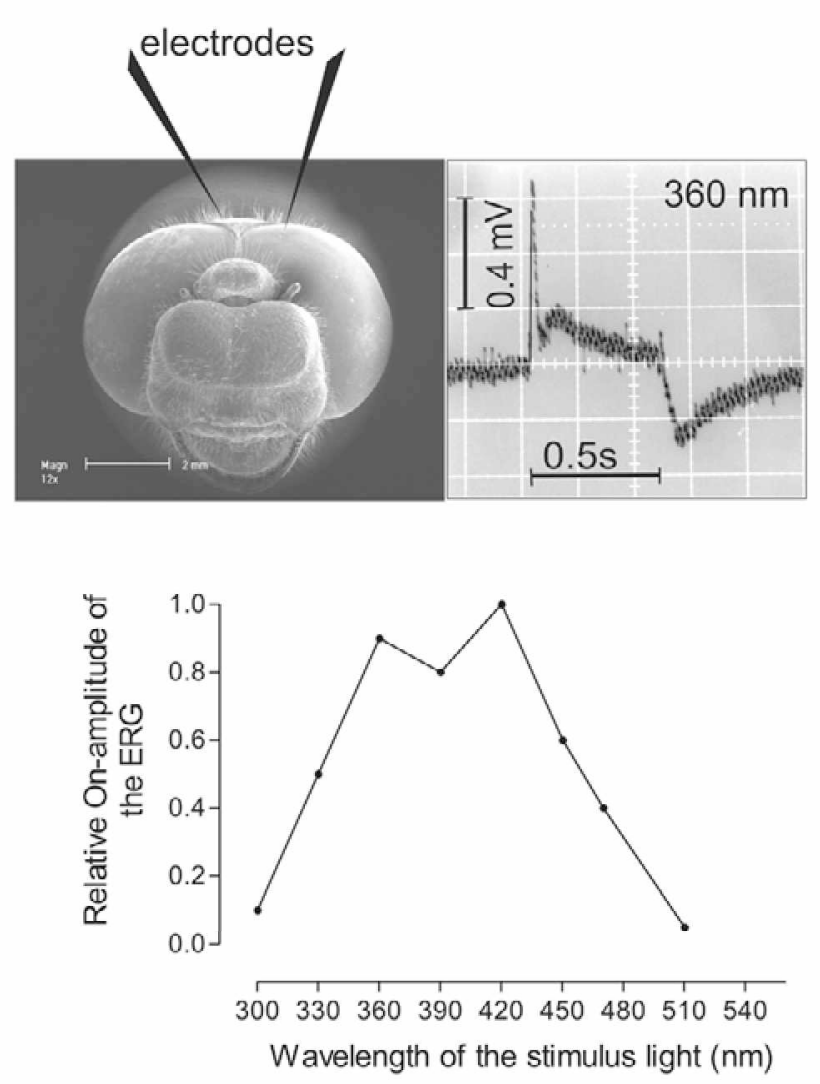

Fig. 2. Spectral sensitivity of the dorsal fovea of the compound eye of Libellula quadrimaculata. Inset: ERG recording of one animal.

so that the head and thorax were immobile but the abdomen could move freely. The experiments were carried out during the day at room temperature $\left(21-23^{\circ} \mathrm{C}\right)$, over a period of two to three hours. Using micromanipulators, tungsten electrodes (sharpened electrolytically in aqueous $\mathrm{NaNO}_{2} / \mathrm{KOH}$ solution, with tips $10-20 \mu \mathrm{m}$ ) were inserted subcorneally into the dorsal part of the compound eye (recording electrode) and into the haemolymph space of the vertex of the head (indifferent electrode) (Fig. 2). ERG responses elicited with light stimuli from a $150 \mathrm{~W}$ xenon arc lamp were recorded via a photosensitive diod, preamplified, and displayed in DC mode on a dual beam storage oscilloscope. For monochromatic stimulation, white stimulus light was passed through one of 14 narrow-band interference filters, using filter wheels ranging from 300 to $700 \mathrm{~nm}$ (Oriel Instruments). The monochromatic stimuli were presented in a different random sequence at 3-min intervals, and a reference stimulus of white light was given at the beginning and the end of the test stimuli to control for measurement reliability. The duration of each stimulus was $0.5 \mathrm{sec}$. With such short stimuli, undesired light-induced motion of the screening pigment, which could have an uncontrolled influence on the measurement results, could probably be ruled out. For each test stimulus, the amplitude of the On-transient of the ERG was measured (Fig. 2). The largest amplitude was defined as $100 \%$ and served as the reference value (see also Kral \& Stelzl, 1998).

Determination of the short-wave radiation from the sky and calculation of the foveal response. To determine the section of the sky viewed by the dorsal fovea of the compound eyes of the perching dragonfly, the following measurements were used: (i) the angle from the zenith $\left(0^{\circ}\right)$ to the sun, i.e. the solar zenith angle (sza), (ii) the difference between the yaw angle $\phi$ of the perching male and the solar azimuth $\varepsilon$, i.e. the viewer azimuth angle $\theta$ ( Fig. 1a), and (iii) the vertical viewing angle of the dorsal fovea $\left(\beta+90^{\circ}\right)$ relative to the zenith, i.e. the viewer zenith angle (vza) ( Fig. 1b). With the aid of SBDART (Santa Barbara DISORT Atmospheric Radiative Transfer), a FORTRAN program, the relative radiation values in the UV to blue range from scattered light (not from direct sunlight) were calculated for the whole sky and for the section of the sky viewed by the dorsal fovea of the eye. (For further information, see also Möller, 2002.) The spectral sensitivity values for the dorsal fovea, obtained from the ERG measurements, were first multiplied by the spectral radiation found at the viewing site of the dorsal fovea on the sky (determined from the "mid latitude summer" atmospheric model) and then integrated over the wavelengths. This yielded the foveal response value (FR) at the viewing site on the sky. The maximum foveal response value $\left(\mathrm{FR}_{\max }\right)$ was determined in the same way, but for all lines of sight (for the whole hemisphere of the sky). The relative foveal response value (RFR), i.e. the relative response of the foveal photoreceptors, is the quotient of foveal response value/maximum foveal response value $\left(R F R=F R / F R_{\max }\right)$.

\section{RESULTS}

\section{Perching site and perching behaviour}

Mature Libellula quadrimaculata males appeared at the research pond as early as 0800 hours and alighted on a reed stem, always facing upwards with the wings held open. Individual animals consistently returned to the same perches for more than 3 weeks. The stem was located at the edge of the open pond. The males assumed one of two basic attitudes, either a resting attitude or a foraging attitude, i.e. a sit-and-wait ambush posture. It was possible to differentiate between the two attitudes by means of the head position, since in the resting attitude the head drooped downwards, whereas during active foraging the head was directed forwards (see Miller, 1995). In the present study only data for males engaged in a sit-and-wait ambush posture were analysed. During the course of the measurements the males exhibited typical percher's foraging flights consisting of a sudden takeoff and return to the same perch. Approximately two-thirds of the measurements of perching orientations were made in the morning and one-third in the afternoon. Most of the measurements were made around noon. The mean difference between the yaw angle $(\phi)$ of the perching male and the solar azimuth $(\varepsilon)$, i.e., the mean viewer azimuth angle, $\theta$, was $126.5^{\circ} \pm 43.3^{\circ}$ (S.D., $\mathrm{n}=203, \mathrm{~N}=8$; Fig. 1a). The mean vertical viewing angle of the dorsal fovea $\left(\beta+90^{\circ}\right)$ relative to the zenith, i.e. the mean viewer zenith angle (vza), was $10.9^{\circ} \pm 5.7^{\circ}$ (S.D., $\mathrm{n}=203, \mathrm{~N}=8$; Fig. 1b).

\section{View of the dorsal fovea of the perching dragonfly}

The angular values indicate that the perching males tended to look away from the sun, and that the dorsal fovea was always directed toward the sky. In no case did the dragonfly face directly into the sun. There was not a single case where the fovea was directed below the landscape horizon, i.e. toward vegetation.

Spectral sensitivity of the dorsal fovea. The spectral sensitivity of the dorsal fovea determined from the ERG measurements is shown in Fig. 2. The ERG measure- 


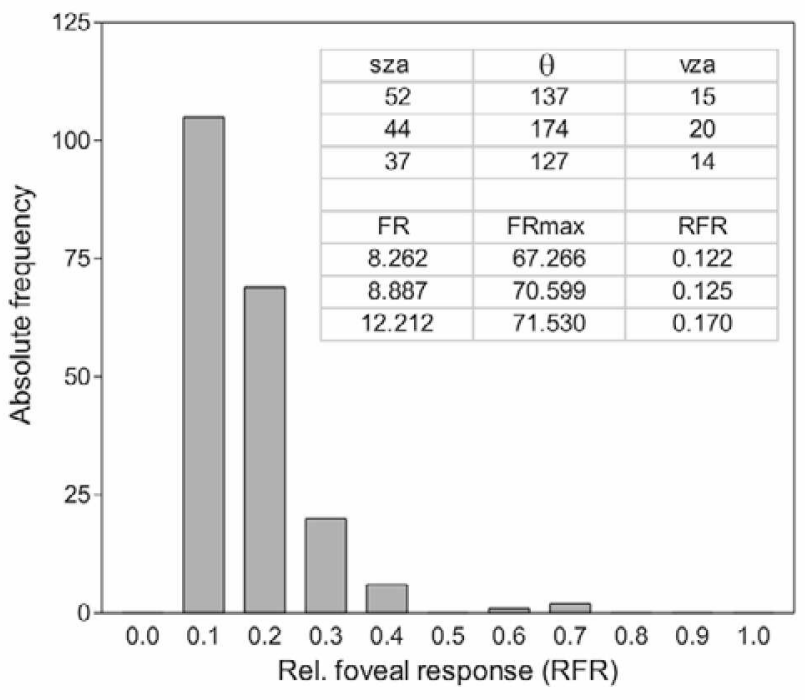

Fig. 3. Histogram illustrating the frequency of the calculated relative foveal responses (RFR) of perching males in terms of the spectral sensitivity of the receptors to the short-wave radiation in the section of the sky viewed by the fovea. F-Test; $\mathrm{R}^{2}=$ $0.97 ; \mathrm{n}=203 ; \mathrm{N}=8$. Inset shows an example of the raw data that were used for calculations; sza, solar zenith angle; $\theta$, viewer azimuth angle; vza, viewer zenith angle; FR, foveal response at the viewing site; $\mathbf{F R}_{\max }$, maximum foveal response for all lines of sight; RFR, relative foveal response, the quotient of foveal response value/maximum foveal response value $\left(\mathrm{FR} / \mathrm{FR}_{\max }\right)$.

ments indicate two response peaks, one of 0.9 in the UV region, at $360 \mathrm{~nm}$, and one of 1.0 in the violet region, at $420 \mathrm{~nm}$.

Short-wave radiation from the sky and foveal response. The sections of the sky viewed by the dorsal fovea of the perching dragonfly are associated with foveal response, calculated from the ERG measurements and radiation values, of between 0.1 and 0.19 in $52 \%$ of cases, between 0.2 and 0.29 in $34 \%$ of cases, and between 0.3 and 0.39 in $10 \%$ of cases (Fig. 3). Thus in almost twothirds of the cases, the dorsal fovea viewed a section of the sky which triggered a relatively low foveal response (RFR).

\section{DISCUSSION}

The diurnal perching orientation of $L$. quadrimaculata males results in a favourable field of view for the dorsal fovea. This field of view is in fact, as expected for functional reasons, based on the characteristics of the fovea (Labhart \& Nilsson, 1995), and is directed toward the blue sky. Of the 203 observations that we made, there was not a single case in which the fovea was directed below the landscape horizon, i.e. toward vegetation. As determined by the perching behaviour, the foveal field of view is not close to the sun, where there is maximum radiation not only of long-wave but also of short-wave light from the sky. The dorsal fovea preferentially views a section of the sky away from the sun, where there is less radiation but a higher blue-violet and UV saturation; i.e. the proportion of blue-violet and UV is higher relative to longer wave radiation than in the section of sky closer to the sun. The fovea of the perching dragonfly is thus directed toward a section of sky which appears the bluest to the human observer. For the perching dragonfly, in this situation the sky should appear bright and uniform, and background features such as foliage should appear relatively indistinct. (The opposite would be true if the dragonfly looked toward the sun). This could be important for the perching dragonfly, because it can detect small flying prey objects better against a motionless, uniform and bright background (see Gogala, 1967; Kral, 2002). Typical prey primarily includes small Diptera, as well as some Zygoptera and Tipulidae, as indicated by the authors' own behavioural studies.

In the case of other prey-detecting insects, such as mantids, moving stimuli that are brighter than the visual background cannot be detected; a stimulus must be darker than its background to be classified as prey (Prete, 1999). This makes sense for the following reason. The moving objects will be perceived as luminance decrements, i.e. as darker than the average background luminance. This could also be expected to be the case here, due to the spectral characteristics of the background viewed by the perching dragonflies (see also Olberg et al., 2000). This is supported by the electrophysiological behaviour of preydetecting neurones (descending contralateral movement detectors, DCMDs) in the mantid brain (Gonka et al., 1999) and target selective descending neurones (TSDNs) in the dragonfly brain (Frye \& Olberg 1995; also see Olberg, 1986). These neurones show a dramatic and significant preference for small black objects being moved in front of a bright background.

The fact that the fovea is directed toward a section of the sky which has a relatively low amount of radiation but a high UV and blue-violet saturation could indicate that relative spectral contrast plays a role. A precondition would be a high amount of UV and low amount of visible (VIS) radiation in the section of sky viewed, in addition a low amount of UV and a high amount of VIS radiation reflected by the prey item. The separation could then occur via a threshold operation measuring UV/VIS contrast. However this would require a comparison between the UV and the VIS receptor; in the case of the dragonfly fovea the VIS receptor corresponds to the blue receptor (Möller, 2002). A precondition would be that the insect prey is illuminated directly by the sun. The direct illumination contains a higher proportion of visible radiation, i.e., with regard to a blue/UV contrast, the background would be relatively strongly illuminated with UV and the insect would appear relatively "blue", assuming that the insect reflected all wavelengths equally. In order to address this question, exact information concerning the chitin reflectivity of the various insect prey would be required. At present these data are lacking.

Relative spectral contrast could also play a role in terms of interspecific recognition of individuals. In order to avoid losing essential resources L. quadrimaculata must promptly detect the males of other dragonfly species, such as Libellula depressa, as they fly into the territory. 
L. depressa males are characteristically black ventrally. Thus there is a particularly strong contrast between the sky and the intruding dragonfly, similar to the situation for prey detection. If the intruder is seen from a different angle, for instance if the intruding male executes a body roll movement on flying into the territory, Libellula quadrimaculata could recognise the intruder on the basis of its bright blue waxy layer, using the blue receptors found in the foveal region. Further optical and electrophysiological investigations of $L$. quadrimaculata are planned.

ACKNOWLEDGEMENTS. We would like to thank Dr. Ralf Möller (Max Planck Institute for Psychological Research, Munich) for the calculation of the spectral radiation values, and for many helpful suggestions. We are also grateful to the reviewers for many valuable comments on the manuscript. In addition, we would like to thank Dr. Edith Stabentheiner for the scanning electron micrographs. Many thanks to Mary Ansell for translation work. This study was supported by the Austrian Science Foundation (Grant No. P14697-Bio to K.K.).

\section{REFERENCES}

Armett-Kibel C. \& Meinertzhagen I.A. 1983: Structural organization of the ommatidium in the ventral compound eye of the dragonfly Sympetrum. J. Comp. Physiol. A 151: 285-295.

BelYshev B.F. 1967: Weather conditions and daily activity of dragonflies (Odonata). Entomol. Rev. 46: 457-460.

EgGENREICH U. \& KRAL K. 1990: External design and field of view of the compound eyes in a raptorial neuropteran insect Mantispa styriaca. J. Exp. Biol. 148: 353-365.

FrYe M.A. \& Olberg R.M. 1995: Visual receptive field properties of feature detecting neurons in the dragonfly. J. Comp. Physiol. A 177: 569-576.

Gogala M. 1967: Die spektrale Empfindlichkeit der Doppelaugen von Ascalaphus macaronius Scop. (Neuroptera: Ascalaphidae). Z. Vergl. Physiol. 57: 232-243.

Gonka M.D., Laurie T.J. \& Prete F.R. 1999: Responses of movement sensitive descending visual interneurons to preylike stimuli in the praying mantis, Sphodromantis lineola (Burmeister). Brain, Behav., Evol. 54: 243-262.

HORRIDGE G.A. 1978: The separation of visual axes in apposition compound eyes. Phil. Trans. R. Soc. Lond. B 285: 1-59.

KraL K. 1987: Organization of the first optic neuropile (or lamina) in different insect species. In: Gupta A.P. (ed.): Arthropod Brain: Its Evolution, Development, Structure, and Functions. John Wiley \& Sons, New York, pp. 181-201.

KrAL K. 2002: Ultraviolet vision in European owlflies (Neuroptera: Ascalaphidae): a critical review. Eur. J. Entomol. 99: $1-4$.
Kral K. \& Stelzl M. 1998: Daily visual sensitivity pattern in the green lacewing Chrysoperla carnea (Neuroptera: Chrysopidae). Eur. J. Entomol. 95: 327-333.

LABHART T. \& NilsSON D.-E. 1995: The dorsal eye of the dragonfly Sympetrum: specializations for prey detection against the blue sky. J. Comp. Physiol. A 176: 437-453.

LAND M.F. 1989: Variations in the structure and design of compound eyes. In: Stavenga D.G. \& Hardie R.C. (eds): Facets of Vision. Springer, Berlin, Heidelberg, New York, pp. 90-111.

LAND M.F. 1997: Visual acuity in insects. Annu. Rev. Entomol. 8: $147-177$.

LaUGHLin S. \& McGinNES S. 1978: The structures of dorsal and ventral regions of a dragonfly retina. Cell Tissue Res. 188: 427-447.

MazoKhin-PoRshnyakov G.A. 1959: Colorimetric study of colour vision in the dragonfly. Biofizika 4: 427-436.

Meinertzhagen I.A., Menzel R. \& Kahle G. 1983: The identification of spectral receptor types in the retina and lamina of the dragonfly Sympetrum rubicundulum. J. Comp. Physiol. A 151: 295-310.

MeYer E.P. \& LABHART T. 1993: Morphological specializations of dorsal rim ommatidia in the compound eye of dragonflies and damselflies (Odonata). Cell Tissue Res. 272: 17-22.

Miller P.L. 1995: Visually controlled head movements in perched anisopteran dragonflies. Odonatol. 24: 301-310.

MöLLER R. 2002: Insects could exploit UV-green contrast for landmark navigation. J. Theor. Biol. 214: 619-631.

Olberg R.M. 1986: Identified target-selective visual interneurons descending from the dragonfly brain. J. Comp. Physiol. A 159: $827-840$.

Olberg R.M., Worthington A.H. \& Venator K.R. 2000: Prey pursuit and interception in dragonflies. J. Comp. Physiol. A 186: $155-162$.

Prete F.R. 1999: Prey recognition. In: Prete F.R., Wells H., Wells P. \& Hurd L.E. (eds): The Praying Mantids. Johns Hopkins Univ. Press, Baltimore, pp. 141-179.

SHERK T.E. 1978: Development of the compound eyes of dragonflies (Odonata). III. Adult compound eyes. J. Exp. Zool. 203: $61-80$.

StavenGa D.G. 1989: Pigments in compound eyes. In: Stavenga D.G. \& Hardie R.C. (eds): Facets of Vision. Springer, Berlin, Heidelberg, New York, pp. 153-172.

WeHNER R. 1981: Spatial vision in arthropods. In: Autrum H. (ed.): Handbook of Sensory Physiology, Vol. VII/6A. Springer, Berlin, Heidelberg, New York, pp. 287-616.

YANG E.-C. \& OSORIO D. 1991: Spectral sensitivities of photoreceptors and lamina monopolar cells in the dragonfly, Hemicordulia tau. J. Comp. Physiol. A 169: 663-669.

Received February 28, 2003; revised June 17, 2003; accepted June 26, 2003 